\title{
High-resolution Manometry for Oropharyngeal Dysphagia in a Patient With Large Cervical Osteophytes
}

\author{
Tae Hee Lee and Joon Seong Lee* \\ Institute for Digestive Research, Soonchunhyang College of Medicine, Seoul, Korea
}

A 61-year-old man presented with difficult swallow. His symptoms began several months ago with progression during past months. He had particular problems in swallowing pills, dry food and meat. His dysphagia localized to neck, without associated odynophagia, nasal regurgitation and cough. He had no other significant medical history. Physical examination revealed unremarkable findings.
Localization to neck is not specific for an oropharyngeal dysphagia, because about $30 \%$ of patients with distal esophageal obstruction perceive the obstruction to be in the cervical esophagus. ${ }^{1}$ Therefore we performed high-resolution manometry (HRM; ManoScan, Sierra Scientific Instruments, Los Angeles, CA, USA) for the evaluation of difficult swallow. HRM showed normal esophageal peristalsis. However, when compared with a
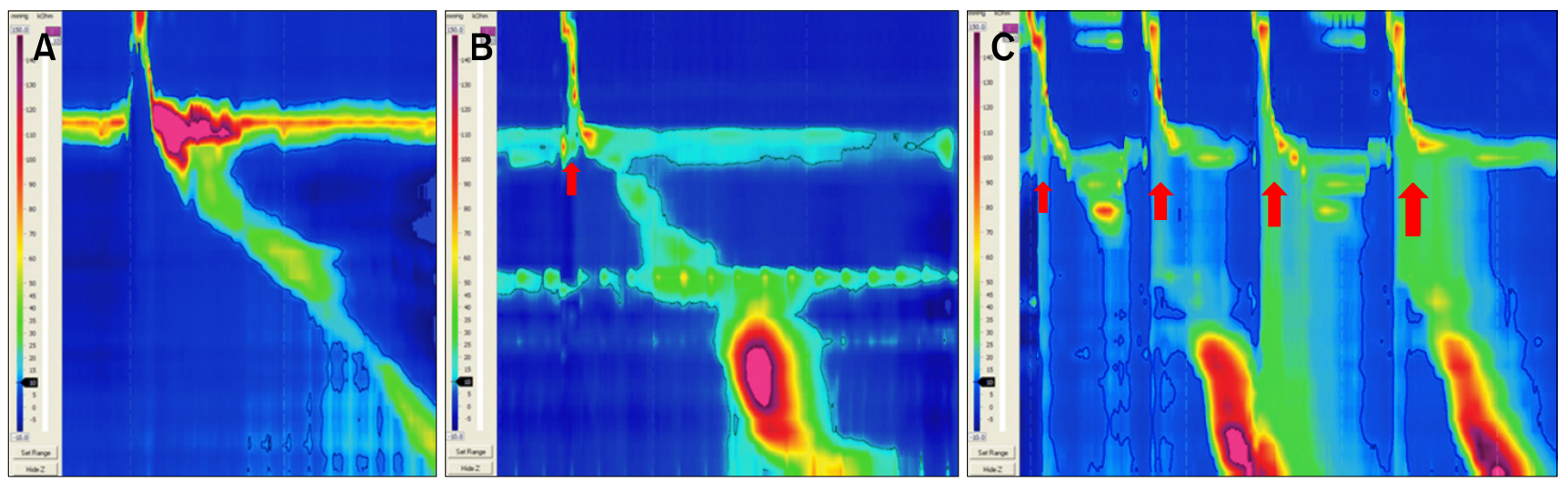

Figure 1. High-resolution manometry focusing on upper esophageal sphincter (UES) during $5 \mathrm{~mL}$ water swallows. (A) UES pressure topography in a healthy 61-year-old man. (B) UES pressure topography in the patient with short sphincter relaxation and elevated intrabolus pressure (red arrow) proximal to UES. (C) Worsening change of the elevated intrabolus pressure (red arrows) during multiple rapid swallows using $100 \mathrm{~mL}$ water.

Received: April 20, 2012 Revised: May 10, 2012 Accepted: May 11, 2012

(c) This is an Open Access article distributed under the terms of the Creative Commons Attribution Non-Commercial License (http://creativecommons. org/licenses/by-nc/3.0) which permits unrestricted non-commercial use, distribution, and reproduction in any medium, provided the original work is properly cited.

*Correspondence: Joon Seong Lee, MD

Institute for Digestive Research, Soonchunhyang College of Medicine, Daesagwan-gil 22, Yongsan-gu, Seoul 140-743, Korea

Tel: +82-2-709-9691, Fax: +82-2-709-9696, E-mail: drjslee@dreamwiz.com

Financial support: None.

Conflicts of interest: None. 




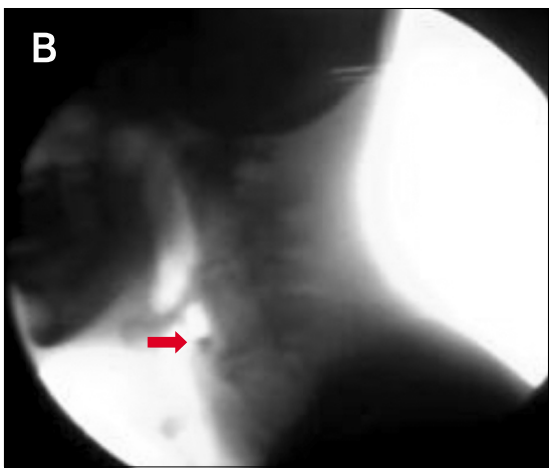

Figure 2. Neck computed tomography (CT) and fluoroscopic view of the patient. (A) Neck CT demonstrates prominent cervical osteophytes including $\mathrm{C} 5$ and C6. (B) Fluoroscopy reveals moderate amount of pharyngeal residue (red arrow) due to reduced upper esophageal sphincter opening, which is at the level of $\mathrm{C} 5$ and $\mathrm{C} 6$. healthy 61-year-old subject (Fig. 1A), there were shorter upper esophageal sphincter (UES) relaxation and elevated intrabolus pressure proximal to UES in the patient (Fig. 1B). Interestingly, worsening change of the elevated intrabolus pressure was noted during multiple rapid swallow using $100 \mathrm{~mL}$ water (Fig. 1C). This finding suggested cricopharyngeal achalasia. We performed further neck CT to evaluate a cause of cricopharyngeal achalasia. Neck CT showed prominent cervical osteophytes including C5 and C6 (Fig. 2A). A further videofluoroscopic swallowing study demonstrated reduced UES opening and moderate amount of pharyngeal residue (Fig. 2B). Given together, the patient had elevated intrabolus pressure gradient across the cervical osteo- phytes. Cervical osteophytes usually are clinically silent but can cause dysphagia, stridor, neurologic problems, and even aspiration when they are larger than $10 \mathrm{~mm}$. $^{2}$ They are seen mainly in older patients.

\section{References}

1. Hirano I, Kahrilas PJ. A 78-year-old man with difficulty swallowing. Clin Gastroenterol Hepatol 2011;9:470-474.

2. Strasser G, Schima W, Schober E, Pokieser P, Kaider A, Denk DM Cervical osteophytes impinging on the pharynx: importance of size and concurrent disorders for development of aspiration. AJR Am J Roentgenol 2000;174:449-453. 